\title{
The journey from disorder to order: transformation of ferrite magnets investigated in situ by combined Bragg \& total scattering analysis
}

\author{
P. Shyam, H. Vijayan, and M. Christensen \\ Department of Chemistry and Interdisciplinary Nanoscience Center (iNANO), Aarhus University, Langelandsgade 140, 8000 Aarhus \\ C, Denmark \\ priyank@inano.au.dk
}

Permanent magnets are key enablers driving the $21^{\text {st }}$ century's diverse electromagnetic technologies [1]. Ceramic magnets based on iron oxides are corrosion resistant and cost-effective permanent magnetic materials with minimal ecological footprint, compared to their lanthanide-based counterparts (such as $\mathrm{SmCo}_{5} ; \mathrm{Nd}_{2} \mathrm{Fe}_{14} \mathrm{~B}$ etc.) [1,2]. Among the ceramic magnets, the hexaferrites $\left(\mathrm{AFe}_{12} \mathrm{O}_{19} ; \mathrm{A}\right.$ $=\mathrm{Ba} / \mathrm{Sr}$ ) constitute the bulk of industrially produced permanent magnets [3,4]. A permanent magnet's properties emerge hierarchically and are influenced by the structure on various length scales. From atomic interactions in the unit cell $(\sim 1-10 \AA)$, through the interactions between crystallites/domains $(\sim 10 \mathrm{~nm}-1 \mu \mathrm{m})$, to the consolidated macroscopic forms $(\sim 0.1 \mathrm{~mm}-0.1 \mathrm{~m})$, magnetism evolves over length scales spanning 10 orders of magnitude [5]! For maximal magnetic performance, the structure of a permanent magnet must be optimized at all levels. Control over the structure is obtained by controlling the synthesis and processing parameters. Additionally, these fabrication methods need to be economical, effective and compatible with industrial scale synthesis processes.

Recent work from our group has demonstrated the fabrication of high-performance nanostructured $\mathrm{SrFe}_{12} \mathrm{O}_{19}$ hexaferrite permanent magnets from solid-state processing of ferrihydrite - a poorly crystalline, structurally disordered nanomaterial [6,7]. This fabrication method is low-cost, scalable and compatible with industry processes. In the presence of $\mathrm{Sr}^{2+}$ ions, ferrihydrite is seen to act as a building block for the $\mathrm{SrFe}_{12} \mathrm{O}_{19}$ hexaferrite, irrespective of synthesis technique (hydrothermal/microwave/solid-state synthesis) [7-9]. While the transformation of ferrihydrite to hexaferrite has been documented in previous studies, a detailed understanding of the conversion process in situ is lacking. The fundamental question that remains to be answered: how does long-range crystalline order in the hexaferrite magnet arise from the nanoscale-disordered ferrihydrite precursor?

Here, we report on our efforts towards addressing this question using in situ synchrotron X-ray scattering studies to investigate the ferrihydrite-hexaferrite transformation. Ferrihydrite samples (with $\mathrm{Sr}^{2+}$ ) were heated while scattering data was collected in situ at the P02.1 beamline at PETRA III. The P02.1 beamline allows for 2 operating modes separately optimized for X-ray Bragg scattering and total scattering[10]. In situ X-ray scattering experiments were performed in both modes. Combining Rietveld modelling of Bragg scattering data and Pair Distribution Function (PDF) modelling of total scattering data provided detailed insight into the real-time structural evolution of disordered ferrihydrite to crystalline hexaferrite. PDF analysis shed light on the evolution of the short- and intermediate-range structural features during the transformation. Rietveld analysis helped ascertain the long-range order, nanoscale morphology and crystallite growth mechanism. This clear picture of the ferrihydrite-hexaferrite transformation over multiple relevant length scales is expected to aid future efforts in engineering better permanent magnets. Meanwhile, beyond permanent magnetic materials, these insights are also expected to contribute toward a broader understanding of the evolution of crystalline order from nanoscale disorder.

[1] Gutfleisch, O., Willard, M. A., Brück, E., Chen, C. H., Sankar, S. G., \& Liu, J. P. (2011) Adv. Mater. $23,821$.

[2] Sugimoto, M. (2010) J. Am. Ceram. Soc. 82, 269.

[3] Pullar, R. C. (2012) Prog. Mater. Sci. 57, 1191.

[4] de Julian Fernandez, C., Sangregorio, C., de la Figuera, J., Belec, B., Makovec, D., \& Quesada, A. (2020) J. Phys. D. Appl. Phys. 54, 153001 .

[5] Skomski, R. (2003) J. Phys. Condens. Matter. 15, R841.

[6] Christensen, M. \& Vijayan, H. Enhanced magnetic properties through alignment of non-magnetic constituents (2020) P5698EP00-CLI 2020.

[7] Vijayan, H., Knudsen, C. G., Mørch, M. I., \& Christensen, M. (2021) Mater. Chem. Front. 5, 3699.

[8] Granados-Miralles, C., Saura-Múzquiz, M., Bøjesen, E. D., Jensen, K. M. Ø., Andersen, H. L., \& Christensen, M. (2016) J. Mater. Chem. C. 4, 10903.

[9] Grindi, B., BenAli, A., Magen, C., \& Viau, G. (2018) J. Solid State Chem. 264, 124.

[10] Dippel, A.-C., Liermann, H.-P., Delitz, J. T., Walter, P., Schulte-Schrepping, H., Seeck, O. H., et al. (2015) J. Synchrotron Radiat. 22 , 675.

\section{Keywords: Nanomaterials; Total scattering; Bragg scattering; Ferrihydrite; Permanent magnets}

